\title{
Healing of Skin Wounds in Rats: Influence of Low-Level Laser Therapy, Alone or Combined with Natural Products
}

\author{
Diana Milanez Ávila Dias Maciel, Sheila Canevese Rahal, Ivan Felismino Charas dos Santos, \\ Carlos Eduardo Fonseca Alves, , Alexandre Botelho de Abreu Sampaio, Laís Rosa Nagai, \\ Barbara Wagner Duarte Ferraz de Camargo \& Gabriella Cunha Salewski
}

\begin{abstract}
Background: A great variety of natural products have been evaluated for the skin wound healing due to anti-inflammatory, antioxidant and antibacterial activities, and procollagen synthesis, of which may be mentioned the honey and propolis. In turn, low-level laser therapy (LLLT) is considered an important tool in the treatment of skin wounds, because of ability to raise the ATP production, to stimulate the microcirculation, and formation of new blood vessels. Therefore, this study aimed to assess the influence of LLLT, alone or combined with natural products, in the healing of excisional skin wounds in rats. Materials, Methods \& Results: One hundred twenty-six male Wistar rats, aged 14 weeks, were randomly divided into seven groups ( $\mathrm{n}=18$ per group) according to wound treatments: G1 (negative control): 0.9\% saline solution; G2 (positive control): allantoin + zinc oxide ointment; G3: LLLT; G4: mixture of honey $+5 \%$ propolis hydroalcoholic solution; G5: LLLT + mixture of honey + hydroalcoholic solution of 5\% propolis; G6: LLLT + honey in natura; G7: LLLT + 5\% propolis hydroalcoholic solution. Six rats from each group were euthanized on the 7th, 14th, and 21 th postoperative days. Macroscopic and histological evaluations of wound healing were performed. A decrease in wound area occurred in all groups, but in general G4 had the higher reduction and G1 and G3 had the lower reductions. These findings corresponded to the percentage of wound contraction, since the largest contraction was G4 and the lowest contractions were G1 and G3. Histological analysis showed no significant difference among groups on the 7th postoperative day. On postoperative day 14, a significant decrease in hemorrhage occurred between G1xG4, G1xG5 and G1xG7, and a significant decrease in congestion between G1xG7 and G2xG5. In addition, intensity of the inflammatory infiltrate showed a significant reduction between $\mathrm{G} 1 \mathrm{xG} 7, \mathrm{G} 2 \mathrm{xG7}$ and $\mathrm{G} 3 \mathrm{xG} 7$. On postoperative day 21, a significant changed from mixed to mononuclear inflammatory infiltrate was observed between G1xG3, G1xG4, G1xG6, G1xG7, G2xG3, and G2xG6. Regarding organization of fibroblasts, there were differences between G1xG6 and G1xG7.

Discussion: Spite the red laser has been associated to in increase fibroblast proliferation, G3 showed inadequate decrease in wound area and percentage of contraction. Histological examination showed no differences among groups in the inflammatory phase. However, in the proliferative and remodeling phases around days 14 and 21 were found some differences among groups, which may be related to the actions of the natural products, or laser. Studies have shown that honey acts as a repair and anti-inflammatory agent in skin wound that may be associated to lower hemorrhage in G4 and G4 compared to G1 on day 14. Propolis is related to reduction of free radicals and increasing the amount of collagen, which may have contributed for quality improvement of the inflammatory infiltrate in G4 and G7 compared to G1, and fibroblast organization in G7 at postoperative day 21. In conclusion, macroscopically the mixture of honey $+5 \%$ propolis hydroalcoholic solution was the most effective in reducing wound area and increasing wound contraction. However, based on the parameters evaluated histologically, may be highlighted the evolution of the treatments with LLLT + honey, as well as LLLT + $5 \%$ propolis hydroalcoholic solution.
\end{abstract}

Keywords: honey, propolis, biomodulation, wound, healing. 


\section{INTRODUÇÃO}

Vários produtos naturais têm sido avaliados no tratamento de feridas cutâneas, devido as propriedades anti-inflamatórias, antioxidante, antibacterianas, e síntese pró-colágeno [10,11,23]. Entre os produtos apícolas podem ser citados o mel e a própolis $[7,10,15,16,18,23]$.

O mel é um produto viscoso que contém mais de 200 substâncias, tendo como composto primário o açúcar [16]. O mesmo parece ter capacidade de erradicar a infecção sem efeitos adversos; promover redução da inflamação, inchaço, dor e odor; bem como estimular o processo cicatricial da ferida $[10,15,19]$, sendo efetivo no tratamento de vários tipos de feridas cutâneas [18]. Mais de 300 constituintes têm sido identificados em amostras de própolis, porém as atividades biológicas são atribuídas especialmente a presença de flavonoides, ácidos fenólicos e compostos aromáticos $[7,21,23]$. Já foi demonstrada a capacidade da própolis em acelerar a cicatrização de feridas, inclusive em modelos animais que mimetizam as feridas diabética [7,21,22].

Por sua vez, a terapia laser de baixa intensidade (TLBI) é considerada uma importante ferramenta no tratamento de feridas cutâneas, com capacidade de atuar em vários níveis no processo de regeneração $[1,24]$. O tipo de laser utilizado, potência e duração da aplicação influenciam na ação sobre tecido e resultado [13,24]. Outra opção que tem sido proposta é a combinação da bioestimulação laser e tratamentos farmacológicos em feridas com condições de cicatrização enfraquecida [2].

Neste sentido, o presente estudo teve por objetivo avaliar a influência da TLBI, isolada ou combinada com produtos naturais, na cicatrização de feridas cutâneas induzidas por modelo excisional em ratos.

\section{MATERIAIS E MÉTODOS}

\section{Aquisição das substâncias e laserterapia}

Foram utilizados o mel in natura e o raspado de própolis na forma bruta do setor de apicultura da Faculdade de Medicina Veterinária e Zootecnia (UNESP, Botucatu). Antes do processamento, realizou-se a cultura e isolamento bacteriano, no qual foi identificado Enterobacter cloacae e E. aerogenes na amostra de raspado de própolis. A amostra de mel apresentou cultivo bacteriano negativo.
A preparação da solução hidroalcoólica de própolis a 5\% foi realizada com a mistura do raspado da própolis na sua forma bruta com álcool (70\%) e o extrato etanoico foi diluído em água. Para a elaboração da mistura de mel e própolis, foram utilizados $2 \mathrm{~mL}$ da solução hidroalcoólica de própolis a $5 \%$ para cada $10 \mathrm{~g}$ de mel. O mel foi utilizado na forma in natura. Após o processamento, realizou-se novamente o exame microbiológico da mistura, que se apresentou negativo. Para a realização da laserterapia utilizou-se laser de baixa intensidade (laser RECOVER) ${ }^{1}$ com dose de 50J/ $\mathrm{cm}^{2}$, potência $=100 \mathrm{~mW}$, tempo de $25 \mathrm{~s}$.

Animais e ambiente de experimentação

Foram utilizados 126 ratos hígidos da espécie Rattus novergicus da linhagem Wistar, heterogenéticos, machos inteiros, com idade de 14 semanas, e massa corpórea entre 300 e 400 g. Os ratos foram alocados individualmente em caixas plásticas e receberam água e ração comercial para ratos ad libitum. O ambiente era climatizado, com controle de umidade e de luz (ciclos de $12 \mathrm{~h}$ claro/escuro), temperatura de $22-25^{\circ}$ C e umidade entre $40-60 \%$ e as caixas foram limpas a cada 48 h. O período de adaptação ao ambiente experimental foi de 10 dias.

\section{Procedimento experimental}

Os ratos foram submetidos à pesagem e o protocolo anestésico foi realizado com associação de xilazina $(10 \mathrm{mg} / \mathrm{kg})$ e cloridrato de cetamina $(75 \mathrm{mg} /$ $\mathrm{kg}$ ) por via intraperitoneal. Posteriormente, realizou-se tricotomia da região torácica dorsal, de aproximadamente $6 \mathrm{~cm}^{2}$ e desinfecção com clorexidina (2\%) da mesma área. Feridas cutâneas de $2 \mathrm{~cm}$ de diâmetro foram realizadas com auxílio de um "punch" metálico. A pele e gordura subcutânea foram removidos com auxílio de tesoura Metzembaum e pinça anatômica, ambas estéreis. Preservou-se a fáscia muscular dorsal. A hemostasia foi realizada pelo método de compressão digital, utilizando-se gaze esterilizada.

Os ratos foram alocados, aleatoriamente, utilizando o programa Randomizer ${ }^{\circledR}$, em sete grupos de 18 animais, de acordo com o tratamento da ferida: Grupo 1 (controle negativo): solução salina $(0,9 \%)$; Grupo 2 (controle positivo): pomada cicatrizante à base de alantoína + óxido de zinco $\left(\text { Alantol }^{\circledR}\right)^{2}$; Grupo 3: TLBI; Grupo 4: mistura de mel + solução hidroalcoólica de própolis a 5\%; Grupo 5: TLBI + mistura de mel + solução hidroalcoólica de própolis a 5\%; Grupo 6: TLBI + 
mel in natura; Grupo 7: TLBI + solução hidroalcoólica de própolis a $5 \%$.

O tratamento foi realizado a cada $24 \mathrm{~h}$ e as feridas foram limpas com gaze estéril umedecida em solução salina $(0,9 \%)$, antes da aplicação das substâncias e uso do laser de baixa intensidade. A mistura de mel e própolis e o mel in natura foram aplicados com auxílio de uma espátula de madeira estéril. A solução hidroalcoólica de própolis a 5\% foi aplicada por meio de conta-gotas estéril. A TLBI foi realizada por ponto.

As feridas permaneceram sem curativo durante todo o período de estudo. Realizou-se, também, a observação do estado geral dos ratos e da ingestão de alimentos e água; avaliação macroscópica das feridas; aferição da temperatura retal e pesagem dos ratos. Durante o estudo, não foi aplicado nenhum antibiótico.

A avaliação macroscópica foi realizada de acordo com a presença de hemorragia, presença de crosta, odor, presença de edema e/ou pus e formação do tecido de granulação. Todos os animais tiveram suas lesões fotografadas por câmera digital durante todos os dias do experimento para posterior avaliação da área e contração da ferida, usando o programa Image ${ }^{\circledR}$. Os tratamentos e as avaliações foram realizados por dois avaliadores independentes e os dados, anotados em ficha própria e individual para cada rato.

Seis animais de cada grupo foram submetidos às biopsias excisionais após eutanásia com tiopental sódico $(120 \mathrm{mg} / \mathrm{kg})$ associado com lidocaína sem vasoconstritor $(7 \mathrm{mg} / \mathrm{kg}$ ) por via intraperitoneal. As eutanásias foram realizadas em três momentos: ao sétimo dia, ao $14^{\circ}$ dia e ao $21^{\circ}$ dia após o início do tratamento.

As feridas foram excisadas com margem de 1 $\mathrm{cm}$ de pele íntegra em torno da lesão, em profundidade até a fáscia muscular. As amostras foram colocadas em frascos contendo formaldeído $10 \%$ tamponado por $24 \mathrm{~h}$ e, posteriormente, mantidas em álcool $70 \%$ até a inclusão em parafina. Cortes de $4 \mu \mathrm{m}$ foram obtidos, sendo os fragmentos corados pelos métodos de hematoxilina-eosina (HE). Foram avaliadas as camadas dérmicas, presença de células de reação inflamatória e diferenciação celular. As lâminas foram observadas no microscópio ótico, com objetivas de 10x e 40x, e as imagens registradas por fotomicrografia. Para a análise estatística as variáveis hemorragia, congestão e quantidade de infiltrado inflamatório foram classificadas com um escore semi-quantitativo de acordo com a intensidade em leve (grau 1), moderada (grau 2) e intensa (grau 3). Para as variáveis úlcera e atividade de fibroblastos foi considerado como presente ou ausente. No caso do infiltrado inflamatório este foi qualificado como mononuclear ou misto (células mononucleares e polimorfonucleares). Quanto à organização tecidual, classificou-se como organizado ou desorganizado.

Swabs estéreis das feridas foram coletados no quinto e no sétimo dia após o início do tratamento, e os mesmos foram enviados para cultura e isolamento bacteriano.

\section{Análise estatística}

Para análise estatística dos dados macroscópicos em cada um dos grupos foi empregada a análise de variância (ANOVA) para amostras repetidas, com contrastes pelo método de Tukey e teste de Kolmogorov-Smirnov. Para avaliação entre momentos foi usado teste de Friedman para as variáveis que não obedeceram às características da normalidade. Para comparação entre os sete grupos, em cada momento avaliado (7, 14 e 21 dias), empregou-se a análise de variância para amostras independentes (ANOVA One way) para dados normais, e teste de Kruskal-Wallis para variáveis fora das condições da normalidade. Diferenças foram consideradas significativas $\operatorname{com} P<0,05$. Com relação aos dados histológicos empregaram-se os teste qui-quadrado e teste exato de Fisher. Diferenças foram consideradas significativas $\operatorname{com} P<0,05$.

\section{RESULTADOS}

A temperatura corpórea manteve-se dentro dos padrões fisiológicos normais da espécie, variando entre $36,2^{\circ} \mathrm{C}$ e $37,1^{\circ} \mathrm{C}\left(36,7 \pm 0,5^{\circ} \mathrm{C}\right)$. A massa corpórea diminui entre o primeiro e segundo dia após as induções, e aumentou a partir do terceiro dia. Não foi observado nenhum óbito e nem sinais clínicos de infecção. Os swabs estéreis das feridas não apresentaram crescimento bacteriano e fúngico.

\section{Avaliação macroscópica}

Em todas as feridas, não foi observada a presença de odor fétido, edema e/ou pus em todos os ratos ao longo do tratamento.

No sétimo dia de pós-operatório, o aspecto da ferida se associava principalmente ao produto aplicado, que mostrava impregnação no caso dos produtos naturais. No $14^{\circ}$ dia de pós-operatório já se observa a presença de tecido de granulação e epitelização das bordas das feridas. No $21^{\circ}$ dia de pós-operatório, todas 
as feridas estavam parcialmente cicatrizadas, sendo observada uma menor área na tratada com mistura de mel e solução hidroalcoólica de própolis a 5\% (Grupo 4). A Figura 1 ilustra o aspecto macroscópico das feridas.

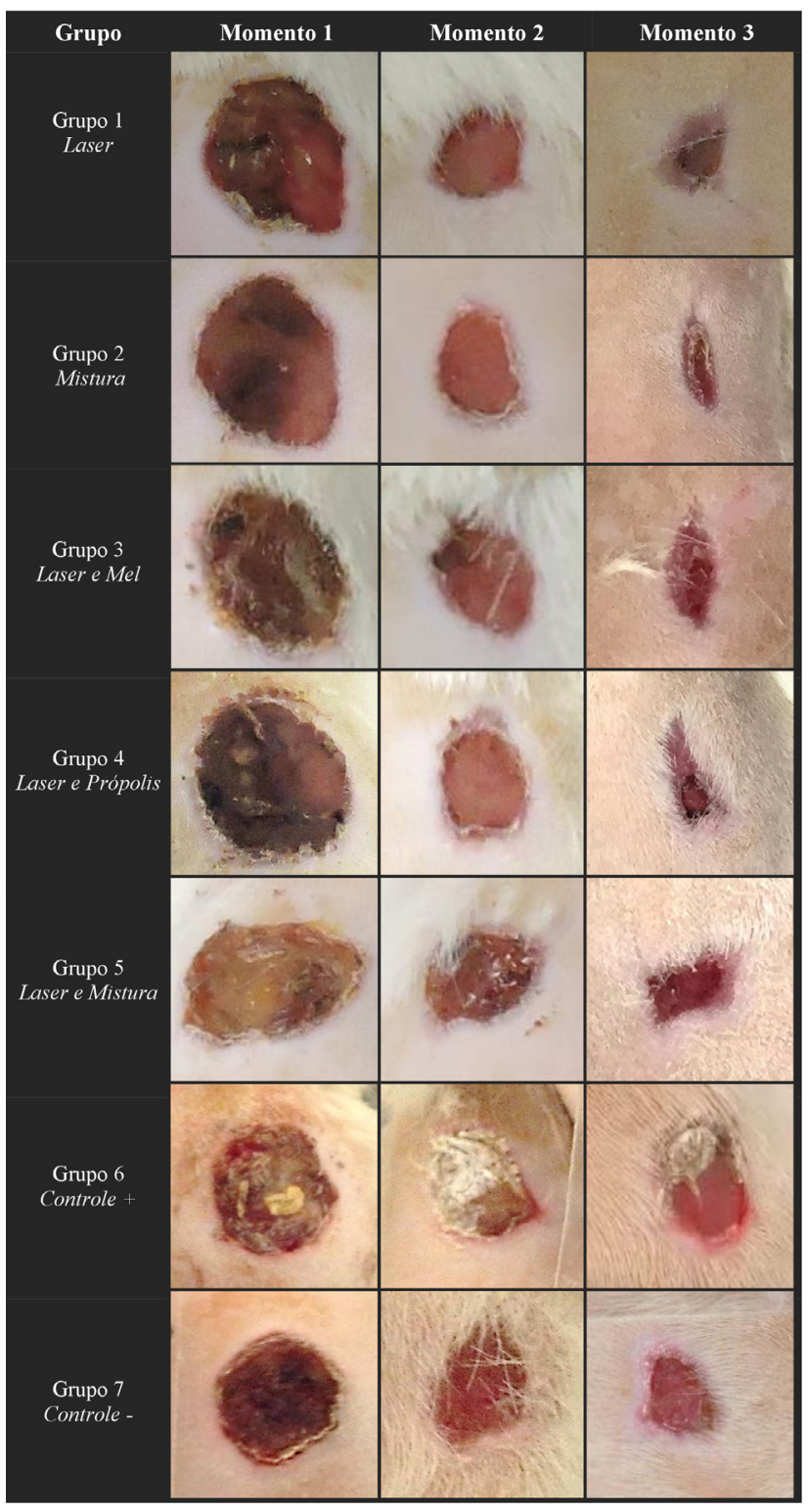

Figura 1. Imagens fotográficas da evolução da cicatrização das feridas tratadas com solução salina $0,9 \%$ (Grupo 1$)$, pomada cicatrizante à base de alantoína e óxido de zinco (Grupo 2), laser de baixa intensidade (Grupo 3), mistura de mel e solução hidroalcoólica de própolis a 5\% (Grupo 4), laser de baixa intensidade e mistura de mel e solução hidroalcoólica de própolis a 5\% (Grupo 5), laser de baixa intensidade e mel in natura (Grupo 6), laser de baixa intensidade e solução hidroalcoólica de própolis a 5\% (Grupo 7), aos sete, 14 e 21 dias de pós-operatório.

\section{Mensuração da área e da contração da ferida}

Em relação às áreas das feridas na comparação do mesmo grupo entre momentos (M1: 7 dias; M2: 14 dias; M3: 21 dias) houve uma diminuição significativa da área da ferida em todos os grupos (Tabela 1), sendo:
Grupo 1 - M1 vs M2 $(\mathrm{P}<0,05), \mathrm{M} 2$ vs M3 $(P$ $<0,001)$, M2 vs M3 $(P<0,01)$

Grupo 2 - M1 vs M2 $(P<0,01)$, M1 vs M3 $(P<0,01)$

Grupo 3 - M1 vs M3 $(P<0,01)$

Grupo 4 - M1 vs M2 $(P<0,01)$, M1 vs M3 $(P$ $<0,01)$, M2 vs M3 (P<0,001)

Grupo 5 - M1 vs M2 (P<0,001), M1 vs M3 $(P<0,001)$

Grupo 6 - M1 vs M2 $(P<0,001)$, M1 vs M3 $(P<0,001)$

Grupo 7 - M1 vs M2 $(P<0,001)$, M1 vs M3 $(P<0,001)$, M2 vs M3 $(P<0,05)$

Por sua vez, na comparação do mesmo momento entre grupos foi observada alteração das áreas das feridas (Tabela 1), sendo:

Momento 1 - G1 vs G4 $(P<0,001)$, G1 vs G7 $(P<0,01)$; G3 vs G5 $(\mathrm{P}<0,01)$; G5 vs G7 $(P<0,01)$

Momento 2 - G1 vs G2 $(P<0,001)$, G1 vs G4 $(P<0,001)$, G1 vs G6 $(P<0,001)$; G3 vs G5 $(P$ $<0,001)$, G3 vs G6 $(P<0,001), \mathrm{G} 3$ vs G7 $(P<0,05)$

Momento 3 - G1 vs G2 $(P<0,05), \mathrm{G} 1$ vs G4 $(P<0,001), \mathrm{G} 1$ vs G5 $(P<0,05), \mathrm{G} 1$ vs G6 $(P<$ 0,01), G1 vs G7 $(P<0,01)$; G2 vs G3 $(P<0,001)$, G2 vs G4 $(P<0,05)$; G3 vs G4 $(P<0,001)$, G3 vs G7 $(P<0,001)$; G4 vs G5 $(P<0,05)$, G5 vs G7 $(P$ $<0,01)$

Em relação às contrações das feridas na comparação do mesmo grupo entre momentos (M1: 7 dias; M2: 14 dias; M3: 21 dias) houve aumento significativo em todos os grupos (Tabela 2), sendo:

Grupo 1 - M1 vs M2 $(P<0,05)$, M2 vs M3 ( $P$ $<0,001)$, M2 vs M3 $(P<0,01)$

Grupo 2 - M1 vs M2 $(P<0,01)$, M1 vs M3 $(P<0,01)$

Grupo 3 - M1 vs M2 $(P<0,05)$, M1 vs M3 $(P<0,01)$

Grupo 4 - M1 vs M2 (P<0,01), M1 vs M3 ( $P$ $<0,01)$, M2 vs M3 $(P<0,001)$

Grupo 5 - M1 vs M2 $(P<0,001)$, M1 vs M3 $(P<0,001)$

Grupo 6 - M1 vs M2 $(P<0,001)$, M1 vs M3 $(P<0,001)$

Grupo 7 - M1 vs M2 $(P<0,001)$, M1 vs M3 $(P<0,001), \mathrm{M} 2$ vs M3 $(P<0,05)$

Por sua vez, na comparação do mesmo momento entre grupos foi observada alteração das contrações das feridas (Tabela 2), sendo: 
Momento 1 - G1 vs G4 $(P<0,01), \mathrm{G} 1$ vs G7 $(P<0,01)$, G4 vs G5 $(P<0,01)$, G5 vs G7 $(P<0,01)$, G4 vs G6 $(P<0,05)$, G6 vs G7 $(P<0,05)$

Momento 2 - G1 vs G2 $(P<0,001), \mathrm{G} 1$ vs G4 $(P<0,001), \mathrm{G} 1$ vs G5 $(P<0,001), \mathrm{G} 1$ vs G6 $(P$ $<0,001)$, G1 vs G7 $(P<0,001)$; G3 vs G5 $(P<0,01)$, G3 vs G6 $(P<0,01)$, G3 vs G7 $(P<0,05)$ Momento 3 - G1 vs G2 $(P<0,05), \mathrm{G} 1$ vs G4 $(P<0,001), \mathrm{G} 1$ vs G5 $(P<0,05), \mathrm{G} 1$ vs G6 $(P<0,01), \mathrm{G} 1$ vs G7 $(P<$ $0,01)$; G2 vs G3 $(P<0,01)$, G2 vs G4 $(P<0,05)$; G3 vs G4 $(P<0,001), \mathrm{G} 3$ vs G5 $(P<0,01), \mathrm{G} 3$ vs G6 $(P$ $<0,001), \mathrm{G} 3$ vs G7 $(P<0,001)$

\section{Exame histopatológico das feridas}

Na comparação entre momentos de cada grupo não foi observada diferença significativa em relação à presença de crosta entre os momentos em nenhum dos grupos avaliados. Contudo, com relação à hemorragia, todos os grupos apresentaram diminuição significativa nos momentos avaliados (M1: 7 dias; M2: 14 dias e M3: 21 dias de pós-operatório):

Grupo 1 - M2xM3 $(P=0,0111)$

Grupo 2 e Grupo 3 - M1xM3 $(P=0,0025)$; M2xM3 (P = 0,0138)

Grupo 4 e Grupo 5 - M1xM3 $(P=0,0025)$

Grupo 6 - M1xM3 $(P=0,0041)$

Grupo 7 - M1xM2 $(P=0,0067)$; M1xM3 $(P$ $=0,0041)$

Em relação à congestão, foi observada diminuição significativa em todos os grupos, excetuando o grupo G1 nos momentos avaliados (M1: 7 dias; M2: 14 dias e M3: 21 dias de pós-operatório):

Grupo 2 - M1xM3 $(P=0,0123)$; M2xM3 ( $P$ $=0,0157$ )

Grupo 3 e Grupo 4 - M1xM3 $(P=0,0025)$

Grupo 5 - M1xM2 $(P=0,0123)$; M1xM3 $(P$ $=0,0111$ )

Grupo 6 - M1xM2 $(P=0,0094)$

Grupo 7 - M1xM2 $(P=0,0094)$; M1xM3 $(P$ $=0,0183$ )

A intensidade do infiltrado inflamatório diminui significamente em todos os grupos nos momentos avaliados (M1: 7 dias; M2: 14 dias e M3: 21 dias de pós-operatório):

Grupo 1 - M1xM3 $(P=0,0025)$

Grupo 2 e Grupo 4 - M1xM2 $(P=0,0138)$; M1xM3 $(P=0,0025)$

Grupo 3 - M1xM3 $(P=0,0025)$; M2xM3 $(P$ $=0,0273)$
Grupo 6 - Grupo 7 - M1xM2 $(P=0,0025)$; M2xM3 $(P=0,0025)$

Por outro lado, em relação à característica do infiltrado inflamatório ocorreu diferença significativa de infiltrado celular inflamatório misto para infiltrado celular mononuclear somente nos grupos G3 e G6 nos momentos (M1: 7 dias; M2: 14 dias e M3: 21 dias de pós-operatório):

Grupo 3 e Grupo 6 - M1xM3 $(P=0,0152)$; M2xM3 $(P=0,0152)$.

Finalizando, com relação à organização dos fibroblastos foi observada uma diferença significativa de desorganização dos fibroblastos para organização dos fibroblastos somente no grupo G6 nos momentos avaliados (M1: 7 dias; M2: 14 dias e M3: 21 dias de pós-operatório): M1xM3 $(P=0,0152)$ e entre M2xM3 $(P=0,0152)$.

Na comparação entre os grupos no sétimo dia de pós-operatório não foram observadas diferenças significativas em relação à presença de crosta, hemorragia e congestão; intensidade e característica do infiltrado inflamatório; e organização dos fibroblastos.

Com 14 dias de pós-operatório não foram detectadas diferenças estatísticas com relação à presença de crosta, característica de infiltrado inflamatório e organização dos fibroblastos. No entanto, ocorreu diminuição significativa quanto à hemorragia nos seguintes grupos: G1xG4 $(P=0,0273)$, G1xG5 $(P=0,0273)$ e $\mathrm{G} 1 \mathrm{xG7}(P=0,0094)$. Em relação à congestão, foi observada diminuição significativa no grupo G1xG7 $(P=0,0123)$ e $\mathrm{G} 2 \mathrm{xG5}(P=0,0357)$. Além disso, com relação à intensidade do infiltrado inflamatório ocorreu diminuição significativa nos grupos G1xG7 $(P=$ 0,0438), G2xG7 $(P=0,0273)$ e G3xG7 $(P=0,0273)$. Aos 21 dias de pós-operatório não foram detectadas diferenças estatísticas com relação à crosta, hemorragia, congestão e intensidade do infiltrado inflamatório. Em relação às características do infiltrado inflamatório foi notada diferença significativa de infiltrado inflamatório misto para infiltrado inflamatório mononuclear nos seguintes grupos: G1xG3 $(P=0,0034), \mathrm{G} 1 \mathrm{xG} 4(P=$ 0,0143); G1xG6 $(P=0,0034)$; G1xG7 (P= 0,0143); G2xG3 ( $P=0,0209)$; G2xG6 $(P=0,0209)$. Com relação à organização dos fibroblastos ocorreu diferença significativa de desorganização para organização dos fibroblastos nos grupos G1xG6 $(P=0,0034)$ e G1xG7 $(P=0,0143)$.

Nas Figuras 2 e 3 estão ilustrados alguns achados da aparência histológica de feridas cutâneas. 
Tabela 1. Áreas $\left(\mathrm{cm}^{2}\right)$ das feridas induzidas em ratos Wistar e avaliadas aos sete dias (M1), 14 dias (M2) e 21 dias (M3) após o início dos tratamentos com solução salina $0,9 \%$ (G1), pomada cicatrizante à base de alantoína e óxido de zinco (G2), laser de baixa intensidade (G3), mistura de mel e solução hidroalcoólica de própolis a 5\% (G4), laser de baixa intensidade e mistura de mel e solução hidroalcoólica de própolis a 5\% (G5), laser de baixa intensidade e mel in natura (G6), laser de baixa intensidade e solução hidroalcoólica de própolis a 5\% (G7).

\begin{tabular}{cccc}
\hline Grupo & $\begin{array}{c}\text { M1 } \\
\text { (Média } \pm \text { Desvio Padrão) }\end{array}$ & $\begin{array}{c}\text { M2 } \\
\text { (Média } \pm \text { Desvio Padrão) }\end{array}$ & $\begin{array}{c}\text { M3 } \\
\text { (Média } \pm \text { Desvio Padrão) }\end{array}$ \\
\hline G1 & $2,15 \pm 0,37^{\text {Aade }}$ & $1,63 \pm 0,36^{\mathrm{Bac}}$ & $0,92 \pm 0,27^{\mathrm{Ca}}$ \\
G2 & $1,76 \pm 0,68^{\text {Aabcde }}$ & $0,81 \pm 0,29^{\mathrm{Bbcd}}$ & $0,52 \pm 0,26^{\mathrm{Bb}}$ \\
G3 & $1,56 \pm 0,32^{\mathrm{Aac}}$ & $1,23 \pm 0,33^{\mathrm{ABab}}$ & $1,02 \pm 0,24^{\mathrm{Bacef}}$ \\
G4 & $1,14 \pm 0,13^{\mathrm{Abc}}$ & $0,79 \pm 0,22^{\mathrm{Bbcd}}$ & $0,10 \pm 0,04^{\mathrm{Cd}}$ \\
G5 & $2,14 \pm 0,23^{\mathrm{Abe}}$ & $0,57 \pm 0,10^{\mathrm{Bcd}}$ & $0,51 \pm 0,11^{\mathrm{Bbe}}$ \\
G6 & $1,91 \pm 0,27^{\mathrm{Aabc}}$ & $0,55 \pm 0,13^{\mathrm{Bd}}$ & $0,41 \pm 0,12^{\mathrm{BCbdefg}}$ \\
G7 & $1,23 \pm 0,38^{\mathrm{Ac}}$ & $0,71 \pm 0,16^{\mathrm{Bcd}}$ & $0,41 \pm 0,20^{\mathrm{Cg}}$ \\
\hline
\end{tabular}

Médias seguidas de diferentes letras maiúsculas na mesma linha diferem estatisticamente utilizando o Teste Tukey $(P<0,05)$. Médias seguidas de diferentes letras minúsculas na mesma coluna diferem estatisticamente utilizando o Teste Tukey $(P<0,05)$.

Tabela 2. Contração (\%) das feridas induzidas em ratos Wistar e avaliadas aos sete dias (M1), 14 dias (M2) e 21 dias (M3) após o início dos tratamentos com solução salina $0,9 \%$ (G1), pomada cicatrizante à base de alantoína e óxido de zinco (G2), laser de baixa intensidade (G3), mistura de mel e solução hidroalcoólica de própolis a 5\% (G4), laser de baixa intensidade e mistura de mel e solução hidroalcoólica de própolis a 5\% (G5), laser de baixa intensidade e mel in natura (G6), laser de baixa intensidade e solução hidroalcoólica de própolis a 5\% (G7).

\begin{tabular}{cccc}
\hline Grupo & $\begin{array}{c}\text { M1 } \\
\text { (Média } \pm \text { Desvio Padrão) }\end{array}$ & $\begin{array}{c}\text { M2 } \\
\text { (Média } \pm \text { Desvio Padrão) }\end{array}$ & $\begin{array}{c}\text { M3 } \\
\text { (Média } \pm \text { Desvio Padrão) }\end{array}$ \\
\hline G1 & $31,91 \pm 11,7^{\mathrm{Aac}}$ & $48,09 \pm 11,6^{\mathrm{Ba}}$ & $70,62 \pm 8,6^{\mathrm{Ca}}$ \\
G2 & $44,22 \pm 21,7^{\mathrm{Aabc}}$ & $74,36 \pm 9,41^{\mathrm{Bbc}}$ & $83,49 \pm 8,2^{\mathrm{Bc}}$ \\
G3 & $50,67 \pm 9,9^{\mathrm{Aabc}}$ & $62,06 \pm 10,9^{\mathrm{Bac}}$ & $67,43 \pm 7,5^{\mathrm{Bab}}$ \\
G4 & $63,59 \pm 4,3^{\mathrm{Abc}}$ & $74,68 \pm 7,1^{\mathrm{Bbc}}$ & $96,68 \pm 1,5^{\mathrm{Cd}}$ \\
G5 & $31,98 \pm 7,3^{\mathrm{Aab}}$ & $81,95 \pm 3,1^{\mathrm{Bb}}$ & $83,60 \pm 3,8^{\mathrm{Bcd}}$ \\
G6 & $39,07 \pm 8,5^{\mathrm{Aab}}$ & $82,44 \pm 4,2^{\mathrm{Bb}}$ & $87,05 \pm 4,10^{\mathrm{BCcd}}$ \\
G7 & $60,72 \pm 12,1^{\mathrm{Ac}}$ & $77,28 \pm 5,2^{\mathrm{Bb}}$ & $86,78 \pm 6,4^{\mathrm{Ccd}}$ \\
\hline
\end{tabular}

Médias seguidas de diferentes letras maiúsculas na mesma linha diferem estatisticamente utilizando o Teste Tukey $(P<0,05)$. Médias seguidas de diferentes letras minúsculas na mesma coluna diferem estatisticamente utilizando o Teste Tukey $(P<0,05)$.

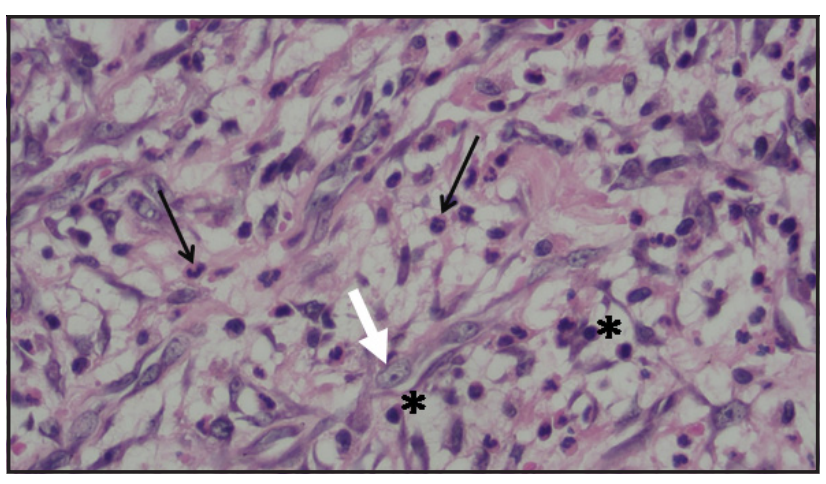

Figura 2. Aparência histológica de ferida cutânea de rato tratada com laser de baixa intensidade e mel in natura (Grupo 6) aos 7 dias de pósoperatório. Observe a presença de fibroblastos ativados (seta branca), células inflamatórias polimorfonucleares (setas pretas) e célula inflamatórias mononucleares (asteriscos) [H\&E; 40X].

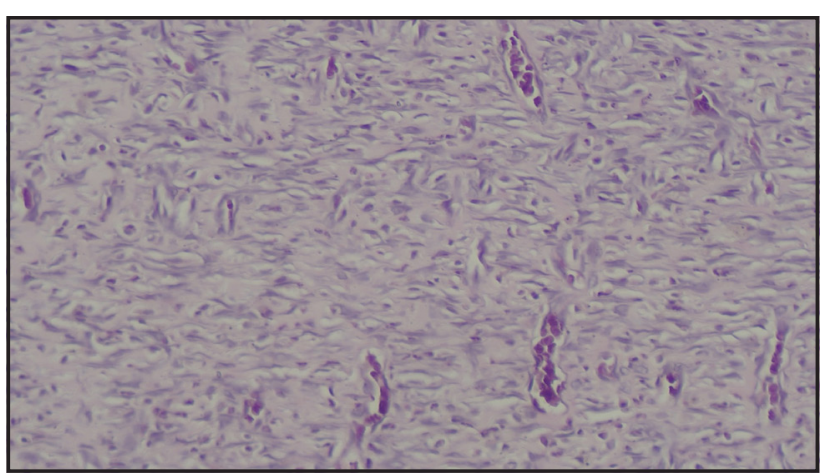

Figura 3. Aparência histológica de ferida cutânea de rato tratada com laser de baixa intensidade e solução hidroalcoólica de própolis a 5\% (Grupo 7) aos 21 dias de pós-operatório. Note a presença de fibroblastos com colágeno em padrão linear [H\&E; 40X]. 


\section{DISCUSSÃO}

Independente do tratamento não ocorreu sinais de infecção na avaliação macroscópica, bem como ocorreu ausência de crescimento bacteriano ou fúngico pelo exame laboratorial de cultura das feridas, confirmando assim que as feridas se mantiveram limpas durante todos os momentos de avaliação.

Ao se avaliar as áreas das feridas no $7^{\circ}$ dia de pós-operatório foi possível verificar que os valores do Grupo 1 controle negativo (solução salina $0,9 \%$ ) se assemelhou ao do Grupo 5 (TLBI + mistura de mel + solução hidroalcoólica de própolis a $5 \%$ ), sendo o menor valor do grupo Grupo 4 (mistura de mel + solução hidroalcoólica de própolis a 5\%), sugerindo que exceto Grupo 5, todos os demais tratamentos atuaram de forma positiva na ferida até este momento. No comparativo entre os três momentos $(7,14,21$ dias) todas as feridas mostraram diminuição da área, sendo a que apresentou melhor evolução foi do Grupo 4 e a pior foi do grupo controle negativo (G1) e grupo G3 (TLBI). Esses mesmos achados coadunaram com a porcentagem de contração, ou seja, a maior contração foi de Grupo 4 e a menor do Grupo 1 e Grupo 3.

Desta forma, macroscopicamente o melhor efeito foi obtido com a associação do mel e própolis (G4). Um estudo com feridas induzidas no pescoço de ratos $(1,5 \times 1,5 \mathrm{~mm})$ também mostrou o efeito sinérgico do mel e própolis em creme, com fase inflamatória mais curta, aumento do tecido de granulação e da angiogênese, quando comparado aos produtos usados de forma isolada [25]. Por sua vez, no $21^{\circ}$ dia de pós-operatório ao se comparar o laser de baixa intensidade com a associação do mel e própolis (G5) nota-se um efeito negativo, mas ainda um pouco melhor comparada ao laser de baixa intensidade e mel (G6) e laser de baixa intensidade e solução hidroalcoólica de própolis (G7).

A fase inflamatória da cicatrização envolve a resposta vascular e as células inflamatórias, notadamente os neutrófilos [4,10], que corresponderia de forma mais intensa ao primeiro momento de avaliação histológica do presente estudo (sétimo dia de pós-operatório), embora tenha que se considerar a sobreposição das fases de cicatrização [10]. Neste momento, não foi possível observar diferenças entre grupos com relação à presença de crosta, hemorragia e congestão; intensidade e característica do infiltrado inflamatório; e organização dos fibroblastos.
Tanto na fase proliferativa como na fase de remodelação [4], na quais estão as avaliações de 14 e 21 dias, já foi possível observar algumas diferenças entre os grupos, o que pode estar relacionado as ações dos produtos naturais empregados, ou do laser.

Vários estudos têm mostrado os efeitos benéficos do mel no tratamento de feridas, tanto como reparador como agente anti-inflamatório [4,5,10,19,21-23]. O mel atua removendo resíduos e bactérias por meio da estimulação dos neutrófilos, macrófagos e fagócitos [21]. Os mecanismos de ação estão relacionados ao peróxido de hidrogênio, alta osmolaridade, acidez, fatores não peróxidos, óxido nítrico e fenóis [21]. Contudo, aos 14 dias de pós-operatório ao se analisar o Grupo 6 (TLBI + mel) este não se distinguiu dos demais, porém nos grupos em que fazia parte da composição, ou seja, Grupo 4 (mistura de mel + solução hidroalcoólica de própolis a 5\%) e Grupo 5 (TLBI + mistura de mel + solução hidroalcoólica de própolis a 5\%), foram observadas menor hemorragia em ambos os grupos em relação ao Grupo 1 (controle negativo) e menor congestão do Grupo 5 comparado ao Grupo 2 (controle positivo). Há também citação que o mel promove ativação do plasminogênio, auxiliando no debridamento autolítico, bem como acelera o desenvolvimento de tecido de granulação saudável [22]. Aos 21 dias de pós-operatório notou-se uma melhora do infiltrado inflamatório do Grupo 4 (mistura de mel + solução hidroalcoólica de própolis a 5\%) em relação ao Grupo 1 (controle negativo), bem como do Grupo 6 (TLBI + mel) em relação ao Grupo 1 (controle negativo) e Grupo 2 (controle positivo). Entretanto, com relação à organização dos fibroblastos foi significativa apenas no Grupo 6 em relação ao Grupo 1.

Importante citar que a produção do mel sofre a ação de fatores ambientais, área geográfica e nutrimentos coletados das plantas, o que pode influenciar na comparação dos resultados entre estudos [10]. Desta forma, a qualidade do mel está associada aos diferentes componentes ativos presentes na composição, incluindo glicose, frutose, flavonoides, polifenóis e ácidos orgânicos [22,23] O mel empregado no atual estudo se inclui na região do Pólo Cuesta do município de Botucatu e já foi caracterizado como de boa qualidade nutricional [20].

Por sua vez, ao se analisar o laser de baixa intensidade e solução hidroalcoólica de própolis a 5\% 
(G7) notou-se aos 14 dias de pós-operatório diminuição da hemorragia e congestão comparado ao Grupo 1 (controle negativo), bem como diminuição do infiltrado inflamatório tanto relativo ao Grupo 1 como Grupo 2 (controle positivo). A própolis é considerada o terceiro componente mais importante produzido das abelhas [22]. Há citações que a fase inflamatória da cicatrização de feridas é reduzida pela própolis devido a redução do número de mastócitos, células inflamatórias e área fibrovascular [21]. Como o mel, a composição da própolis depende das plantas, estação de coleta e espécie de abelha, o que promove heterogeneidade química [26]. O produto empregado no atual estudo tinha como seus principais componentes compostos fenólicos (flavonoides, ácidos aromáticos, benzopiranos), di e triterpenos, e óleos essenciais, entre outros [14]. A própolis auxilia na cicatrização das feridas em virtude da redução da ação de radicais livres; pelas atividades antifúngicas e antibacterianas, associadas a presença de flavonóides, compostos fenólicos, terpenos e enzimas; e por aumentar a quantidade de colágenos tipo I e II, o que auxilia na mais rápida contração [21-23]. Provavelmente essas ações tenham contribuído para que, aos 21 dias de pós-operatório, o Grupo 4 (mistura de mel + solução hidroalcoólica de própolis a 5\%) e o Grupo 7 (laser de baixa intensidade + solução hidroalcoólica de própolis a 5\%) tenham mostrado melhora da qualidade do infiltrado inflamatório comparado ao Grupo 1 (controle negativo), acrescido ainda de maior organização de fibroblastos no Grupo 7.

O laser de baixa intensidade usado no atual estudo foi o arseneto de gálio e alumínio (Ga-Al-As), aplicado na fluência de $50 \mathrm{~J} / \mathrm{cm}^{2}$ e potência de $100 \mathrm{~mW}$. Embora existam várias pesquisas com o emprego da TLBI em feridas em ratos, em análise sistemática foi observado que a despeito da ampla variação de potência (9 a $500 \mathrm{~mW}$ ) e fluência (1 a $60 \mathrm{~J} / \mathrm{cm}^{2}$ ), os melhores resultados foram com a combinação dos comprimentos de onda (vermelho e infravermelho) [13]. O laser vermelho parece atuar nas camadas mais superficiais da ferida e o infravermelho nas mais profundas, sendo que o primeiro parece ser mais eficaz na proliferação de fibroblastos e o segundo na angiogênese [12]. No atual estudo foi usado somente o comprimento de onda $660 \mathrm{~nm}$ (Laser vermelho), o que pode ter influenciado, ao menos macroscopicamente nos resultados. Já que aos 21 dias de pós-operatório a mistura de mel e solução hidroalcoólica de própolis a 5\% (G4) apresentou menor área de ferida comparada à mistura de mel e solução hidroalcoólica de própolis a 5\% e laser (G5), embora sem diferença na porcentagem de contração. Além disso, o Grupo TLBI (G3) não diferiu na área e porcentagem de contração da ferida do grupo solução salina $0,9 \%$ ou grupo controle negativo (G1), aos 21 dias de pós-operatório.

Por outro lado, estudos têm citados efeitos positivos do laser em feridas cutâneas por induzir a proliferação de fibroblastos e células endoteliais, síntese de colágeno, angiogênese e re-epitelização [12], entre outros. Contudo, pela análise histológica do presente estudo, o grupo exclusivamente de TLBI (Grupo 3) atuou principalmente com relação ao infiltrado inflamatório, que foi menor aos 14 dias comparado ao Grupo 7 (TLBI + solução hidroalcoólica de própolis a 5\%) e apresentou melhora da qualidade em relação ao Grupo 2 (controle positivo) aos 21 dias de pós-operatório.

No atual estudo o laser foi aplicado diariamente. Também neste quesito há grande variedade nos estudos com Ga-Al-As em feridas de ratos, com uso diário ou em dias alternados [3,8,9,17]. Contudo, a escolha deve incluir também a fluência e potência do equipamento, sendo a primeira no atual estudo de $50 \mathrm{~J} / \mathrm{cm}^{2}$. Um estudo que avaliou a fluência de 30 $\mathrm{J} / \mathrm{cm} 2$ ou $60 \mathrm{~J} / \mathrm{cm}^{2}$, aplicada diariamente em feridas de $12 \mathrm{~mm}$ em diâmetro induzidas nas costas de ratos, concluiu que a fluência de $60 \mathrm{~J} / \mathrm{cm} 2$ foi a mais efetiva em estimular a angiogênese e maturação do tecido cicatricial, porém o grupo tratado com óleo apresentou maior proliferação de fibroblasto [8]. A condição do tecido é crítica na determinação da dose, já que doses baixas não promovem efeitos biológicos e altas doses podem inibir as funções celulares [6]. Ao se comparar histologicamente os grupos com produto natural e laser, o Grupo 7 (TLBI + solução hidroalcoólica de própolis a 5\%) se distinguiu aos 14 dias de pós-operatório, pela diminuição da hemorragia, congestão e infiltrado inflamatório. Porém, aos 21 dias de pós-operatório, embora a melhora da qualidade do infiltrado inflamatório e organização de fibroblastos tenha sido significativa neste Grupo, o mesmo ocorreu no Grupo 6 (TLBI e mel).

\section{CONCLUSÃO}

Macroscopicamente a mistura de mel e solução hidroalcoólica de própolis a 5\% foi a mais efetiva em 
promover diminuição da área e aumento da contração da ferida. Contudo, baseado nos parâmetros avaliados histologicamente, os grupos TLBI e mel bem como TLBI e solução hidroalcoólica de própolis foram os tratamentos que se destacaram.

\section{MANUFACTURERS}

${ }^{1}$ MM Optics Ltda. São Carlos, SP, Brazil.

${ }^{2}$ Vetnil Ind. e Com. de Produtos Veterinários Ltda. Louveira, SP, Brazil.
Acknowledgements. We thank FAPESP (São Paulo ResearchFoundation) for providing Scientific Initiation Scholarship (number 2017/14098-3). The authors also thank UNIPEX (Unidade de Pesquisa Experimental) - Botucatu.

Ethical approval. This study was approved by the Research Ethics Committee of Botucatu Medical School, UNESP ( $\left.\mathrm{N}^{\circ} 1235 / 2017\right)$.

Declaration of interest. The authors report no conflicts of interest. The authors alone are responsible for the content of the paper.

\section{REFERENCES}

1 Andrade F.S.S.D., Clark R.M \& Ferreira M.L. 2014. Effects of low-level laser therapy on wound healing. Revista do Colégio Brasileiro de Cirurgiões. 41(2): 129-133.

2 Al-Watban F.A.H. \& Andres B.L. 2001. Laser photons and pharmacological treatments in wound healing. Laser Therapy. 12: 1-9.

3 Bayat M., Azari A. \& Galmohammadi M.G. 2010. Effects of 780nm low-level laser therapy with a pulsed gallium aluminum arsenide laser on the healing of a surgically induced open skin wound of rat. Photomedicine and Laser Surgery. 28(4): 465-470.

4 Benhanifia M.B., Boukraâ L., Hammoudi S.M., Sulaiman S.A. \& Manivannan L. 2011. Recent patents on topical application of honey in wound and burn management. Recent Patents on Inflammation \& Allergy Drug Discovery. 5(1): 81-86

5 Bergman A., Yanai J., Weiss J., Bell D. \& David M.P. 1983. Acceleration of wound healing by topical application of honey. American Journal of Surgery. 145(3): 374-376.

6 Chaves M.E., Araújo A.R., Piancastelli A.C. \& Pinotti M. 2014. Effects of low-power light therapy on wound healing: LASER x LED. Anais Brasileiros de Dermatologia. 89(4): 616-623.

7 Garraud O., Hozzein W.N. \& Badr G. 2017. Wound healing: time to look for intelligent, 'natural' immunological approaches? BMC Immunology. 18(Suppl 1): 39-65.

8 Gonçalves R.V., Novaes R.D., Matta S.L.P., Benevides G.P., Faria F.R. \& Pinto M.V.M. 2010. Comparative study of the effects of galliumaluminium- arsenide laser photobiomodulation and healing oil on skin wound in Wistar rats: a histomorphometric study. Photomedicine and Laser Surgery. 28(5): 597-602.

9 Güngörmüs M. \& Akyol U. 2009. The effect of gallium-aluminum arsenide 808nm low-level laser therapy on healing of skin incisions made using a diode laser. Photomedicine and Laser Surgery. 27(6): 895-899.

10 Ibrahim N., Wong S.K., Mohamed I.N., Mohamed N., Chin K.Y., Ima-Nirwana S. \& Shuid A.N. 2018. Wound healing properties of selected natural products. International Journal of Environmental Research and Public Health. 15(11): 1-23.

11 Kotian S., Bhat K., Pai S., Nayak J., Souza A., Gourisheti K. \& Padma D. 2018. The role of natural medicines on wound healing: a biomechanical, histological, biochemical and molecular study. Ethiopian Journal of Science. 28(6): 759-770.

12 Lima F.J.C., Barbosa F.T. \& Sousa-Rodrigues C.F. 2014. Use alone or in combination of red and infrared laser in skin wounds. Journal of Lasers in Medical Sciences. 5(2): 51-57.

13 Lima F.J., Oliveira Neto O.B., Barbosa F.T., Nascimento Galvão A.M., Ramos F.W., Lima C.C. \& Sousa Rodrigues C.F. 2016. Is there a protocol in experimental skin wounds in rats using low-level diode laser therapy (LLDLT) combining or not red and infrared wavelengths? Systematic review. Lasers in Medical Science. 31(4): 779-787.

14 Mani F., Damasceno H.C., Novelli E.L., Martins E.A. \& Sforcin J.M. 2006. Propolis: Effect of different concentrations, extracts and intake period on seric biochemical variables. Journal of Ethnopharmacology. 105(1-2): 95-98.

15 Majtan J. 2014. Honey: An immunomodulator in wound healing. Wound Repair and Regeneration. 22(2): 187-192.

16 Martinotti S. \& Ranzato E. 2018. Honey, wound repair and regenerative medicine. Journal of Functional Biomaterials. 9(2): 1-7.

17 Medrado A.P., Soares A.P., Santos E.T., Reis S.R.A. \& Andrade Z.A. 2008. Influence of laser photobiomodulation upon connective tissue remodeling during wound healing. Journal of Photochemistry and Photobiology B: Biology. 92(3): 144-152.

18 Meo S.A., Al-Asiri S.A., Mahesar A.L. \& Ansari M.J. 2017. Role of honey in modern medicine. Saudi Journal of Biological Sciences. 24(5): 975-978. 
19 Molan P.C. 1999. The role of honey in the management of wounds. Journal of Wound Care. 8(8): 415-418.

20 Ito E.H., Araújo W.L.P., Shinohara A.J., Barros D.C.B., Camilli M.P. \& Orsi R.O. 2018. Physicochemical characteristics of honeys of Apis mellifera L. from the Polo cuesta region, Sao Paulo, Brazil. Boletim de Indústria Animal. 75: 1-9.

21 Oryan A., Alemzadeh E. \& Moshiri A. 2018. Potential role of propolis in wound healing: Biological properties and therapeutic activities. Biomedicine \& Pharmacotherapy. 98: 469-483.

22 Pasupuleti V.R., Sammugam L., Ramesh N. \& Gan S.H. 2017. Honey, propolis, and royal jelly: a comprehensive review of their biological actions and health benefits. Oxidative Medicine and Cellular Longevity. 21p. doi: $10.1155 / 2017 / 1259510$.

23 Pereira R.F. \& Bártolo P.J. 2016. Traditional therapies for skin wound healing. Advances in Wound Care. 5(5): 208229.

24 Rocha Junior A.M., Vieira B.J., Andrade L.C.F. \& Aarestrup F.M. 2007. Effects of low-level laser therapy on the progress of wound healing in humans: the contribution of in vitro and in vivo experimental studies. Jornal Vascular Brasileiro. 6(3): 258-266.

25 Takzaree N., Hadjiakhondi A., Hassanzadeh G., Rouini M.R. \& Manayi A. 2016. Synergistic effect of honey and propolis on cutaneous wound healing in rats. Acta Medica Iranica. 54(4): 233-239.

26 Zabaiou N., Fouache A., Trousson A., Baron S., Zellagui A., Lahouel M. \& Lobaccaro J.A. 2017. Biological properties of propolis extracts: something new from an ancient product. Chemistry and Physics of Lipids. 207(Pt B): 214-222. 